\title{
Buckling of bridging fibres in composites
}

HWAI-CHUNG WU, TAKASHI MATSUMOTO, V. C. LI

Advanced Civil Engineering Materials Research Laboratory, Department of Civil and Environmental Engineering, University of Michigan, Ann Arbor, MI 48109-2125, USA

In brittle materials such as concrete and ceramics, fibre reinforcement has been widely accepted as an effective way of improving their strength and toughness. In addition, a notable pseudo strain-hardening phenomenon can contribute to a significantly enhanced ductility of the composite when an adequately designed fibre system is used. This condition was first proposed by Aveston et al. [1], and later extended by Marshall et al. [2] for continuous aligned fibre reinforced brittle matrix composites. More recently, further extensions to randomly oriented discontinuous fibre reinforced composites have been presented $[3,4]$. Upon satisfying the conditions described in the above mentioned micromechanical models, the ultimate tensile strains of the composites are usually improved by two orders of magnitude [5-7]. Total fracture energy reaching $35 \mathrm{~kJ} / \mathrm{m}^{2}$ was also reported for a $2 \%$ polyethylene fibre reinforced cement paste [8]. This kind of ductile fracture resembles metal instead of brittle materials.

The pseudo strain-hardening behaviour of fibre reinforced brittle matrix composites is associated with multiple cracking, and results from adequate stress transfer capability of bridging fibres. Studies are typically conducted under monotonic tensile loading only. In reality, composites are usually subject to cyclic loads. As a preliminary report of ongoing research on the cyclic behaviour of pseudo strain-hardening cementitious composites, we present initial findings on buckling of bridging nylon fibres across fracture planes in a cement composite after complete unloading in tension. An analytic model is also proposed to account for this buckling phenomenon.

Type I Portland cement, silica fume and superplasticizer were used to form the cement paste with water/cementitious ratio of 0.27 . Discontinuous nylon fibres $\left(L_{\mathrm{f}}=21 \mathrm{~mm}, d_{\mathrm{f}}=25 \mu \mathrm{m}\right.$, and $E_{\mathrm{f}}=5.2 \mathrm{GPa}$ ) were used to reinforce the paste at a volume fraction of $2 \%$. Tensile coupon specimens of size $304.8 \times 76.2 \times 12.7 \mathrm{~mm}$ were prepared and tested under direct tension in a servo hydraulic tester. Detailed mix proportions and testing procedures can be found elsewhere [9]. Tensile stressstrain curves were recorded. An optical microscope with 50 times magnification was used to examine the bridging fibres after the specimen was completely unloaded.

The stress-strain curve is shown in Fig. 1, where four peaks are identified, corresponding to four multiple cracks which occurred within a length of

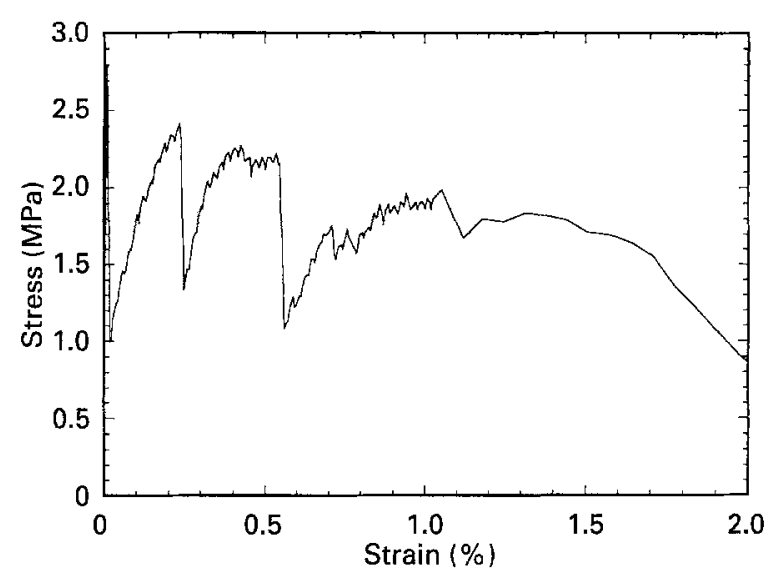

Figure 1 Tensile stress-strain curve of nylon fibre reinforced cement paste $\left(V_{\mathrm{f}}=2 \%\right)$.

$200 \mathrm{~mm}$. After reaching the ultimate load, the main crack opened up continuously with descending load-carrying capacity while other cracks were unloaded. Examination of these closing cracks using a microscope reveals both buckled fibres as well as straight fibres across the crack surfaces. This is shown in Fig. 2. It is clearly demonstrated that a portion of the fibres underwent buckling. In the following, an analysis is made to examine this buckling phenomenon.

It is possible to explain why some of the nylon fibres buckle between the closing crack surfaces by examining whether the critical buckling load of a fibre is exceeded or not. The critical buckling load, $P_{\mathrm{c}}$, for a circular column with both ends fixed is given by

$$
P_{\mathrm{c}}=\frac{\pi^{2} E I}{(0.5 L)^{2}}
$$

where $E=$ Young's modulus of the column, $I=\pi r^{4} / 4, r=$ radius of the column, and $L=$ length of the column [10]. Assuming that the critical buckling load given by the above equation is applicable to a single fibre under compression imposed by the closing crack surfaces, Equation 1 becomes

$$
P_{\mathrm{c}}=\frac{\pi^{2} E_{\mathrm{f}} I}{(0.5 \delta)^{2}}
$$

where $E_{\mathrm{f}}=$ fibre modulus, $I=\pi\left(d_{\mathrm{f}} / 2\right)^{4} / 4, d_{\mathrm{f}}=$ fibre diameter, and $\delta=$ crack opening displacement (cod). This equation yields the relation between critical buckling load $P_{\mathrm{c}}$ and $\operatorname{cod} \delta$ with two fibre parameters included. For a given cod and fibre 


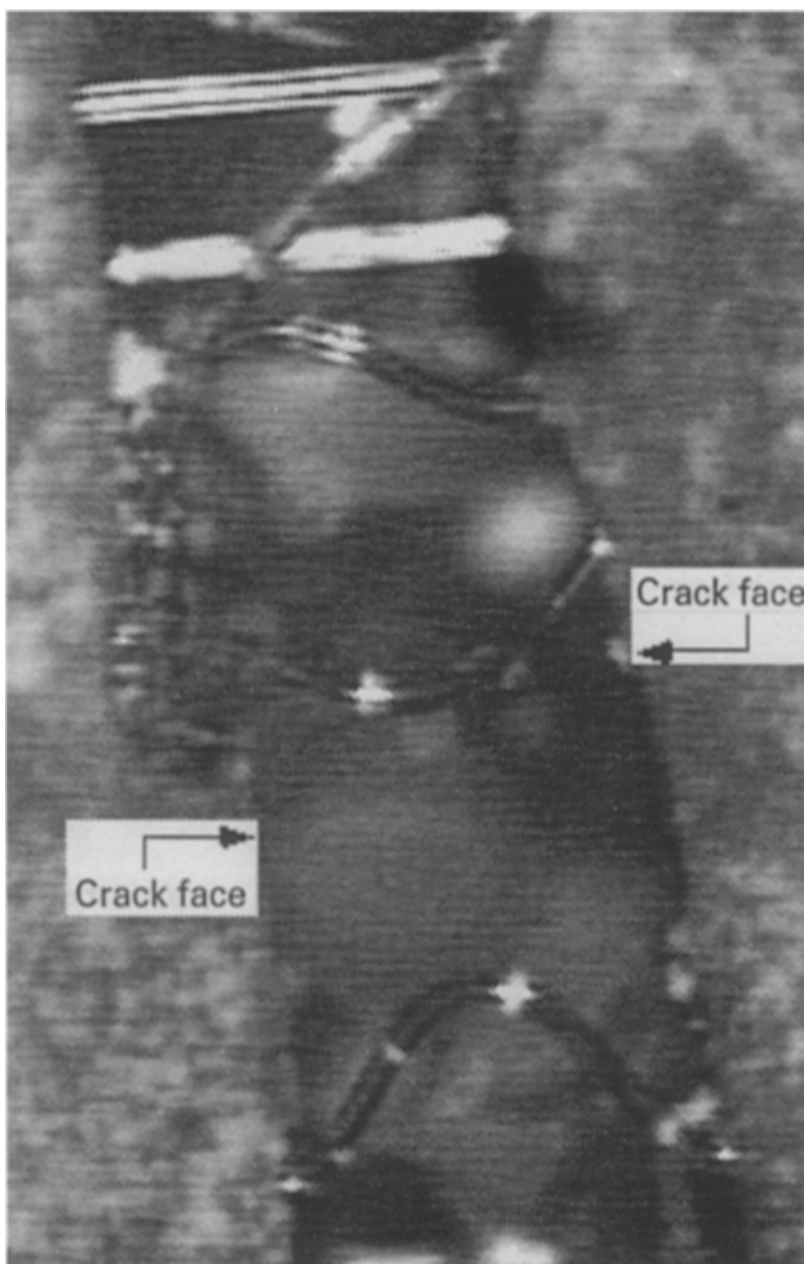

Figure 2 Portion of bridging fibres undergo buckling (computer scanned image).

parameters, $P_{\mathrm{c}}$ can be calculated and compared with axial load applied at the ends of the single fibre. When the axial load exceeds $P_{c}$, the fibre is expected to buckle.

The relation between applied load $P$ and $\operatorname{cod} \delta$ $(P-\delta$ relation) of a single discontinuous fibre bridging a matrix crack with weak (friction controlled) interface has been derived by $\mathrm{Li}$ [11]. The derived $P-\delta$ relation consists of an ascending and descending part, corresponding to debonding and sliding process, respectively. In the ascending part, as $P$ increases, the debonding region extends towards the embedded end of the fibre. During this debonding process the ascending portion of the $P-\delta$ relation is expressed by

$$
P=\frac{\pi}{2} \sqrt{E_{\mathrm{f}} d_{\mathrm{f}}^{3} \tau \delta} \quad \text { for } \delta \leqslant \delta_{0}
$$

where $\tau=$ constant frictional stress and $\delta_{0}=$ $4 \tau l^{2} / E_{\mathrm{f}} d_{\mathrm{f}}$ corresponds to the cod at which frictional debonding is completed and $l=$ embedded length of the fibre. After debonding reaches the embedded end of the fibre, cod is attributed entirely to sliding out of the fibre. In this process $P$ decreases with cod and is given by

$$
P=\pi \tau d_{\mathrm{f}} l\left(1-\frac{\delta-\delta_{0}}{l}\right) \quad \text { for } \delta_{0}<\delta \leqslant l
$$

Applied axial load $P$ imposes a push-back force on the fibre following unloading in tension. Hence the $P-\delta$ relation (originally accounting for tensile load only) needs to be extended to cover compressive load as well. This applied push-back force (in compression) will then be compared with the critical buckling load $P_{\mathrm{c}}$ to determine the possibility of fibre buckling. During crack closure, if the applied push back force $P$ exceeds the critical buckling load, then the fibre will buckle instead of sliding back into the matrix. Derivation of this additional relation $(\Delta P-\Delta \delta$ relation $)$ is described as follows.

Because of random distribution of fibre embedment length at a designated fracture plane in the matrix, fibres can be divided into two groups, i.e. some fibres undergo a debonding process (for long embedment length) and others, sliding (for short embedment length). When unloading occurs $\left(\Delta P=P_{\max }-P_{\min }\right)$, the $\Delta P-\Delta \delta$ relation can be derived by the following equation:

$$
\Delta \delta=\int_{0}^{L_{\mathrm{f}}}\left(\varepsilon_{\max }(x)-\varepsilon_{\text {min }}(x)\right) \mathrm{d} x
$$

where $\Delta \delta=$ cod change, $L_{\mathrm{f}}=$ fibre length, $\varepsilon_{\max }-$ $(x)=$ strain in the fibre at $P=P_{\max }, \varepsilon_{\min }(x)=$ strain in the fibre at $P=P_{\min }$, and $x$ is measured from the shorter embedded end of the fibre (see Fig. 3). The change of cod $\Delta \delta$ between the two crack surfaces results from the difference of axial strain in the fibre and requires summation over the entire fibre length $L_{\mathrm{f}}$. Strain in the fibre $\varepsilon(x)$ is given by

$$
\varepsilon(x)=F(x) /\left(\pi\left(\frac{d_{\mathrm{f}}}{2}\right)^{2} E_{\mathrm{f}}\right)
$$

where $F(x)=$ force in the fibre. Assuming that the interfacial frictional stress $\tau$ is constant in resisting pullout and that unloading creates a zone where $\tau$ acts in the reverse direction to resist unstretching or push-back of the fibre, $F(x)$ becomes a piecewise linear function and so does $\varepsilon(x)$ (movement of the crack surfaces relative to the fibre is assumed to have little effect on cod change). Figs 3 and 4 show the distribution of axial strain of the fibre before and after unloading for fibres in the debonding and sliding process, respectively. As seen in Figs 3 and 4,

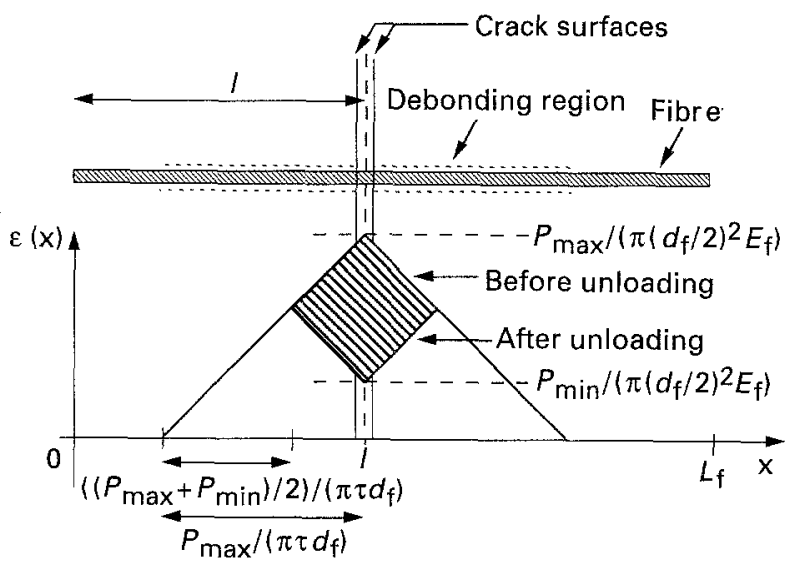

Figure 3 Strain distribution in the debonding fibre with shorter embedment length $l$ before and after unloading. The shaded area shows $\Delta \delta$. 


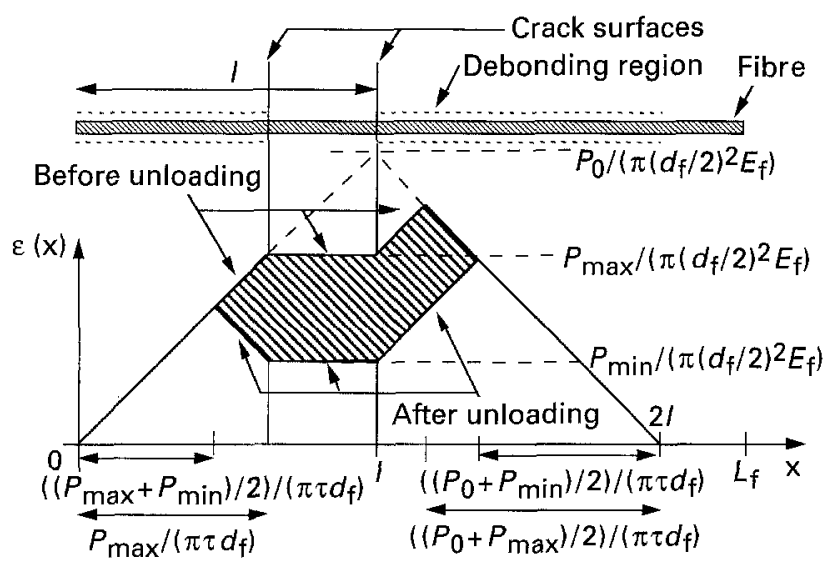

Figure 4 Strain distribution in the sliding fibre with shorter embedment length $l$ before and after unloading. The shaded area shows $\Delta \delta$.

the axial strain arises within the debonded region and the exposed portion of the fibre between the crack surfaces, and equals zero in the undebonded region. Thus the integration in Equation 5 can be carried out only for the debonded region and the exposed portion of the fibre, and is equivalent to computing the shaded area. The shaded area yields the $\Delta P-\Delta \delta$ relation for fibres in debonding and sliding separately. For fibres in debonding

$$
\Delta \delta=\frac{2(\Delta P)^{2}}{\pi^{2} \tau d_{\mathrm{f}}^{3} E_{\mathrm{f}}^{r}}
$$

and for fibres in sliding

$$
\Delta \delta=\frac{1}{\pi^{2} \tau d_{\mathrm{f}}^{3} E_{\mathrm{f}}}\left(2(\Delta P)^{2}+6 \Delta P\left(P_{0}-P_{\max }\right)\right)
$$

where $P_{0}=$ pullout load at which debonding is completed.

These equations hold also for compressive loading. However, for fibres in the sliding stage, $\Delta \delta$ obtained by unstretching or contracting fibre is insufficient to attain full crack closure. At $P_{\min }=-P_{\max }$, the debonded region where interfacial frictional stress acts in the reversed direction reaches the embedded end of the fibre. Further compressive loading beyond $-P_{\max }$ is then required to push the fibre back into the matrix. Therefore Equation $8 a$ is followed by

$$
\Delta \delta=\frac{\Delta P-2 P_{\max }}{\pi \tau d_{\mathrm{f}}}+\frac{1}{\pi^{2} \tau d_{\mathrm{f}}^{3} E_{\mathrm{f}}}\left(12 P_{\max } P_{0}-4 P_{\max }^{2}\right)
$$

for $-P_{0} \leqslant P_{\min } \leqslant-P_{\max }$. During push-back of the fibre, $\Delta \delta$ is attributed mainly to sliding distance, which is represented by the first term of Equation $8 b$, under constant interfacial frictional stress.

Using Equations 3, 4, 7 and $8, P-\delta$ curves including loading and unloading can be constructed, as shown in Fig. 5. For the nylon fibre composite, $P-\delta$ curves are shown in Fig. 6 for various fibre embedment lengths $l$, and for all fibres perpendicular to the fracture surface. Load reversal point is set at $\operatorname{cod}(505 \mu \mathrm{m}$ in this case) which corresponds to the peak load on a fibre with its embedded length equal

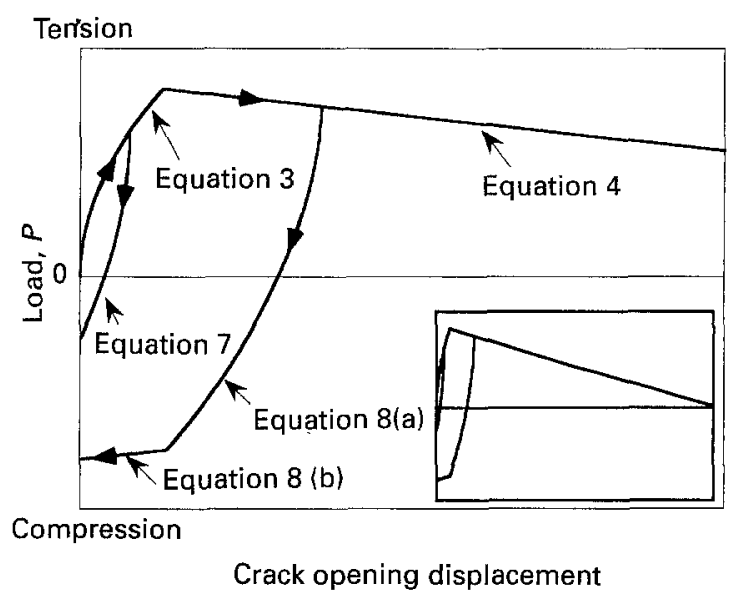

Figure 5 Schematic representation of loading-unloading curves with corresponding governing equations. Insert shows complete curve.

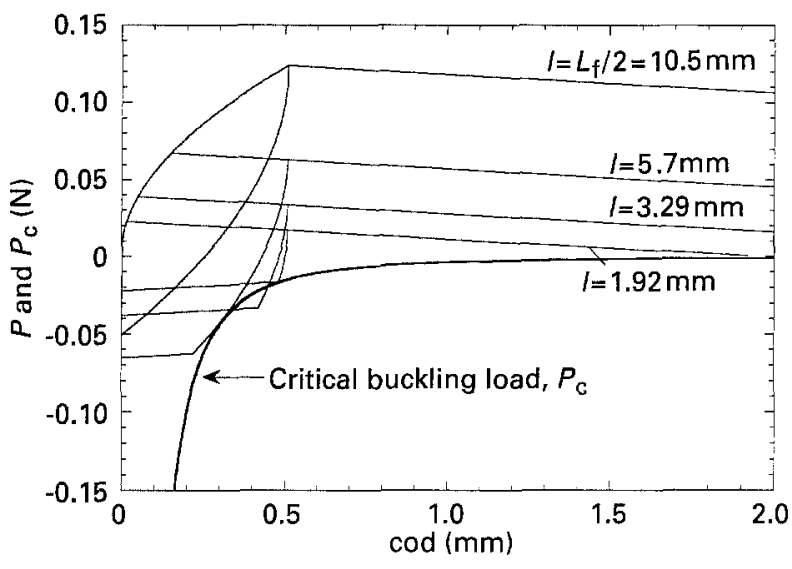

Figure $6 P-\delta$ curves and critical buckling load for nylon fibre perpendicular to the crack plane $\left(\phi=0^{\circ}\right)$.

to $L_{\mathrm{f}} / 2$. This corresponds also to the maximum composite strength $[3,11]$. Material parameters of the composite used are given on the first page of this work and $\tau=0.15 \mathrm{MPa}$ is estimated from the maximum bridging stress. As revealed in Fig. 6 , it is possible for fibres with $1.9 \leqslant l \leqslant 5.7 \mathrm{~mm}$ to buckle, since the critical buckling load is reached for these fibres. It should be noted that this range of fibre embedded lengths for undergoing buckling depends on the location of the load reversal point. In our experiment, unloading occurred following the peak of the composite strength. Hence the load reversal point is chosen as above. Full crack closure can be obtained for fibres with embedded length above $5.7 \mathrm{~mm}$, since the critical buckling load is higher than the necessary compressive load required to push the fibres back into the matrix due to shorter exposed fibre lengths (at the beginning of sliding). On the other hand, maximum contraction load $\left(P_{\min }\right)$ achievable is too small to cause fibre buckling due to shorter embedded length for those fibres with initial embedded length below $1.9 \mathrm{~mm}$. Furthermore there is no chance for the sliding curve to intersect the critical buckling load curve, since the gradient of $P_{\mathrm{c}}$ is much steeper. It should be noted that complete pullout occurs for fibres with initial embedded length less than $505 \mu \mathrm{m}$. 
In addition to the random distribution of fibre embedment length, the angle of a fibre to the crack surface is also distributed randomly in a real composite. It is found that pullout force increases with increasing inclination angle of the fibre to the loading axis $\phi$, and can be accounted for by the snubbing effect $[12,13]$. Under the assumption that the snubbing effect applies to the $\Delta P-\Delta \delta$ relation

$$
\Delta P(\Delta \delta, \phi)=\Delta P(\Delta \delta, \phi=0) \mathrm{e}^{f \phi}
$$

where $f=$ snubbing coefficient $(f=0.994$ for nylon fibre [13]). Figs 7 and 8 have been drawn for $\phi=30^{\circ}$ and $60^{\circ}$. Since the additional force due to the snubbing effect is required for the same amount of

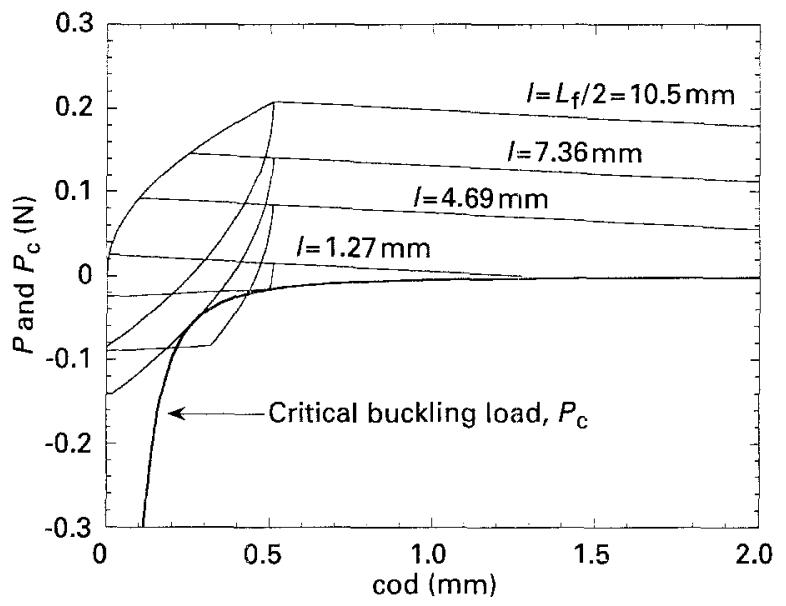

Figure $7 P-\delta$ curves and critical buckling load for nylon fibre inclined at $\phi=30^{\circ}$ to the crack plane.

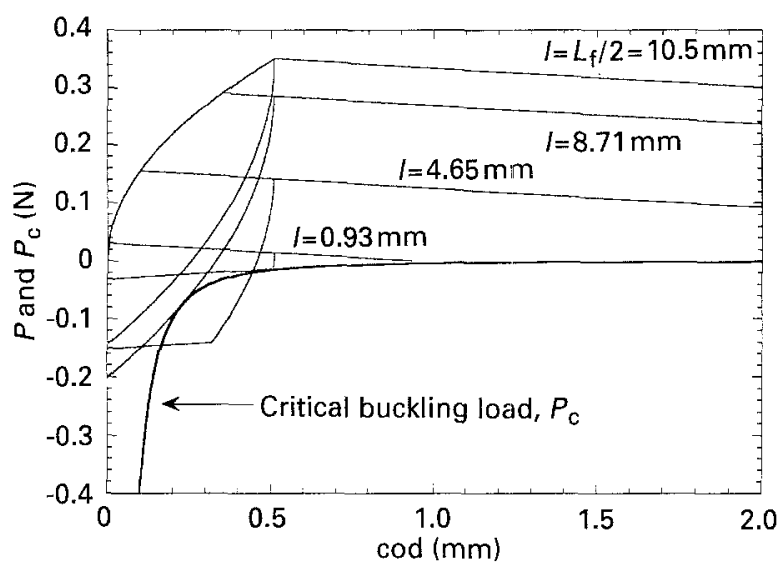

Figure $8 P-\delta$ curves and critical buckling load for nylon fibre inclined at $\phi=60^{\circ}$ to the crack plane. cod change and critical buckling load remains unchanged, more fibres tend to undergo buckling as shown in Figs 7 and 8 . The possible range of fibre embedment length undergoing buckling is 1.3 to $7.4 \mathrm{~mm}$ and 0.9 to $8.7 \mathrm{~mm}$, respectively, for $\phi=30^{\circ}$ and $60^{\circ}$.

It is shown that a portion of the bridging nylon fibres in a cement paste undergo buckling when the specimen is unloaded. This can be accounted for by combining simple buckling theory and fibre debonding and sliding mechanisms in a load-unload cycle. Furthermore, the snubbing effect is shown to enhance fibre buckling.

\section{Acknowledgement}

This work has been supported by a grant from the National Science Foundation (MSS-9301949) to the University of Michigan.

\section{References}

1. J. AVEston, G. A. COOPER and A. KELLY, in Proceedings of the Conference on Properties of Fiber Composites, National Physical Laboratory (Guildford IPC Science and Technology Press, Surrey, 1971) p. 15.

2. D. B. MARShALL, B. N. COX and A. G. EVANS, Acta Metall. 33 (1985) 2013.

3. V. C. LI and C. K. Y. LEUNG, ASCE J. of Engng. Mech. 118 (1992) 2246

4. V. C. LI and H. C. WU, Appl. Mech. Rev. 45 (1992) 390.

5. H. C. WU and V. C. LI, J, Cement Concrete Compos. (1993) (in press).

6. D. B. MARSHALL and B. N. COX, Acta Metall. 35 (1987) 2607.

7. H. KRENCHEL and H. STANG, in Proceedings of the Second International Symposium on Brittle Matrix Composites, 1988, edited by A. M. Brandt and I. H. Marshall (Elsevier Applied Science, London) p. 20.

8. V. C. LI, J. Struct. Mech. Earthquake Engng 471 [I-24] (1993) 37 .

9. V. C. LI, H. C. WU, M. MAALEJ, D. K. MISHRA and T. HASHId A, J. Amer. Ceram. Soc. (1993) (submitted).

10. S. TIMOSHENKO, in "Theory of elastic stability" (McGraw-Hill, New York, 1936) p. 67.

11. V. C. LI, J. Mater. Civ. Engng. 1 (1992) 41.

12. J. MORTON and G. W. GROVES, J. Mater. Sci. 11 (1976) 617.

13. V. C. LI, Y. WANG and S. BACKER, Composites 2 (1990) 132.

Received 22 December 1993

and accepted 4 February 1994 